\title{
Avaliação da buprenorfina pelas vias intravenosa ou intramuscular em cães anestesiados pelo desfluorano
}

\author{
Evaluation of the buprenorphine administered by intravenously or intramuscularly in dogs \\ anesthetized with desflurane
}

\author{
Almir Pereira de Souza ${ }^{1}$ Celina Tie Nishimori ${ }^{1}$ Paulo Sérgio Patto dos Santos ${ }^{2}$ \\ Danielli Parrilha de Paula ${ }^{1}$ Newton Nunes $^{3}$ Márlis Langenegger de Rezende ${ }^{1}$ \\ Piedad Natalia Henao-Guerrero ${ }^{4}$
}

RESUMO

Objetivou-se avaliar comparativamente os efeitos da buprenorfina, administrada pelas vias intramuscular(IM) ou intravenosa (IV), sobre variáveis cardiovasculares, em cães anestesiados com desfluorano. Para tanto, foram utilizados dezesseis cães adultos, clinicamente saudáveis, distribuídos em dois grupos $(n=8)$ denominados de GI e GII. Em ambos os grupos, a anestesia foi induzida com propofol $(8 \mathrm{mg} / \mathrm{kg}, \mathrm{IV})$ e em seguida os animais foram intubados com sonda orotraqueal de Magill, a qual foi conectada ao aparelho de anestesia volátil para administração de desfluorano (1,5 CAM). Após 30 minutos do início da anestesia inalatória, foi aplicado no GI buprenorfina na dose de 0,02 $\mathrm{mg} / \mathrm{kg}$ pela via IV, enquanto no GII administrouse o opióide na mesma dose porém pela via IM. Avaliaram-se: freqüência cardíaca $(F C)$; pressões arteriais sistólica, diastólica e média (PAS, PAD e PAM); débito cardíaco $(D C)$; pressão venosa central $(P V C)$ e pressão da artéria pulmonar (PAP). As colheitas foram feitas nos seguintes momentos: M1 - 30 minutos após o início da anestesia inalatória antes da aplicação do opióide; M2 - 15 minutos após a administração da buprenorfina: M3, M4 e M5 - de 15 em 15 minutos após M2. A avaliação estatística dos dados foi efetuada por meio de Análise de Perfil ( $p<0,05)$ As variáveis PAS, PAM, DC, PVC e PAP, não apresentaram alterações significativas de seus valores em ambos os grupos. Entretanto, a FC e a PAD apresentaram reduções significativas após a administração do opióide apenas no GI. Assim, pôde-se concluir que a buprenorfina administrada pelas vias IV ou IM não interferiu nos índices cardiovasculares de forma a manifestar efeitos clínicos importantes em cães anestesiados com desfluorano.

Palavras-chave: opióides, cardiovascular, anestesia inalatória.

\begin{abstract}
The aim of this study was to evaluate comparatively the effects of buprenorphine administered intramuscularly or intravenously on cardiovascular variables in dogs anesthetized with desflurane. Sixteen adult healthy male and female mongrel dogs were randomly distributed in two groups of eigth animals each (GI and GII). The anesthetic induction was done using propofol (IV), and immediately, the dogs were intubated and submited to desflurane anaesthesia administrated at 1.5 MAC. Thirty minutes after beginning the inhalatory anaesthesia, the GI received buprenorphine $(0.02$ $\mathrm{mg} / \mathrm{kg}$ ) intravenously whereas the GII group received the opioid at the same dose, by IM injection. Heart Rate (HR); Systolic, Diastolic and Mean Arterial Blood Pressure (SAP, $D A P$ and $M A P)$; Cardiac Output (CO); Central Venous Pressure (CVP) and Pulmonar Arterial Pressure (PAP) were evaluated. The measurements were registered 30 minutes after beginning the inhalatory anaesthesia and before opioid administration (M1); 15 minutes after buprenorphine administration (M2). Serial measurements were carried out in 15-minute intervals after the administration of buprenorphine up to 45 minutes (M3, M4 and M5). The numerical data were submited to Profile Analysis $(p<0.05)$. In both groups, SAP, MAP, CO, CVP and PAP values were not significant. However, only in GI, the HR and DAP decreased significantly after opioid administration. The results allow us to conclude that buprenorphine IV or IM did not produce alterations on the cardiovascular parameters that could provide any significant clinics effects in dogs anesthetized with desflurane.
\end{abstract}

Key Words: opioids, cardiovascular, inhalatory anesthesia.

'Doutorando em Cirurgia Veterinária, Faculdade de Ciências Agrárias e Veterinárias (FCAV), Universidade Estadual Paulista (UNESP), Campus de Jaboticabal.

${ }^{2}$ Professor de Anestesiologia Veterinária, Centro Universitário Barão de Mauá, Ribeirão Preto, SP.

${ }^{3}$ Professor, Doutor, Departamento de Clínica e Cirurgia Veterinária. FCAV, UNESP, 14884-900, Jaboticabal, SP. E-mail: newton@fcav.unesp.br. Autor para correspondência.

${ }^{4}$ Mestrando em Cirurgia Veterinária, FCAV, UNESP, Campus de Jaboticabal. 


\section{INTRODUÇÃO}

Nas modernas técnicas de anestesia balanceada, encontram-se com freqüência fármacos opióides seja na fase pré ou trans-operatória, os quais têm como objetivos principais a redução do consumo do anestésico inalatório e promoção de um maior conforto aos pacientes. Tais fármacos podem ser utilizados pelas vias, intravenosa, intramuscular e epidural. Assim, se faz necessário conhecer os efeitos fisiológicos decorrentes do uso destes agentes por tais vias, a fim de melhor selecionar a sua forma de administração.

A buprenorfina é um opióide denominado agonista parcial, ligando-se aos receptores mu os quais são ativados parcialmente (PADDLEFORD, 2001). Possui potência analgésica aproximadamente 30 vezes superior a da morfina (THURMON et al., 1996), aliviando a dor moderada a grave, associada a procedimentos cirúrgicos abdominais, torácicos e ortopédicos (GÓRNIAK, 1996). É um dos opióides com ação analgésica mais longa, com seus efeitos durando de 4 a 8 horas (PADDLEFORD, 2001).

Uma das características hemodinâmicas, conseqüientes da administração deste opióide, em cães, é a redução significativa da freqüência cardíaca, provavelmente por aumento do tono vagal, e mínimas alterações na pressão sangüínea sistêmica (STEPIEN et al., 1995), apresentando, porém, depressão respiratória que pode ser revertida com o uso de antagonistas opióides, como o naloxone (THURMON et al., 1996).

O desfluorano, um anestésico volátil halogenado fluorinado, tem peso molecular igual a 168 , ponto de ebulição aos $23,5^{\circ} \mathrm{C}$, ao nível do mar, pressão de vapor de $664 \mathrm{mmHg}$, a $20^{\circ} \mathrm{C}$ (SMILEY, 1992) e vem sendo utilizado na rotina cirúrgica desde 1992 (WEISKOPF et al., 1992). O baixo coeficiente sangue/gás do desfluorano $(0,42)$ permite rápido aumento ou diminuição da concentração alveolar o que torna rápidas a indução e a recuperação do paciente (EGER, 1992). A administração do desfluorano é associada a aumento da atividade simpática, a qual atinge o pico máximo decorridos 5 minutos de exposição ao fármaco (PACENTINE et al., 1995). A ação simpática deve-se à existência de sítios receptores nas vias aéreas superiores, que respondem rapidamente à elevação da concentração.

No sistema cardiovascular, este anestésico induz queda da pressão arterial média, aumento da freqüência cardíaca (CLARKE et al., 1996), depressão da contratilidade do coração e conseqüente redução do débito cardíaco (PAGEL et al., 1991). O fármaco possui propriedades inibitórias sobre arritmias ventriculares espontâneas, após infarto do miocárdio (NOVALIJA et al., 1998). A administração de desfluorano em cães pré-medicados com a associação fentanil-droperidol bloqueou a bradicardia produzida por tal associação, bem como promoveu retardo na condução elétrica atrial, ventricular e átrio-ventricular caracterizada por alterações nas ondas $\mathrm{P}, \mathrm{QRS}$ e no intervalo PR (SANTOS et al., 2001).

No homem não foram encontradas evidências de que o desfluorano induza isquemia do miocárdio ou de que o fármaco aumente a taxa de mortalidade em pacientes com doenças coronárias (WARLTIER \& PAGEL, 1992). Em coelhos submetidos a infarto experimental, houve marcante diminuição na área afetada e proteção do miocárdio contra injúrias de perfusão (PRECKEL et al., 1998).

Desta forma, objetivou-se, com a realização deste experimento, avaliar as possíveis alterações em algumas variáveis cardiovasculares decorrentes do uso do opióide buprenorfina pelas vias intravenosa ou intramuscular, em cães durante anestesia geral inalatória com desfluorano.

\section{MATERIAL E MÉTODOS}

Foram utilizados dezesseis cães adultos, machos e fêmeas, clinicamente saudáveis, com peso de 11,5kg $( \pm 2,7)$, distribuídos aleatoriamente em dois grupos de igual número, denominados GI e GII. Após jejum alimentar de 12 horas e restrição hídrica de duas horas, os animais foram induzidos à anestesia com $8 \mathrm{mg}$ $\mathrm{kg}^{-1}$ de propofol ${ }^{\mathrm{a}}$, por via intravenosa (IV) e, em seguida, intubados com sonda de Magill. A sonda foi então coaptada ao aparelho de anestesia volátil para administração do desfluorano ${ }^{\mathrm{b}}$, por meio de circuito anestésico tipo "semi-fechado"c, dotado de vaporizador termocompensado, microprocessado e calibrado para o agente anestésico ${ }^{\mathrm{d}}$, tendo como fluxo diluente o oxigênio $\left(30 \mathrm{ml} \mathrm{kg}^{-1} \mathrm{~min}^{-1}\right)$.

O desfluorano foi fornecido na concentração de 1,5 CAM, considerando-se a CAM como sendo equivalente a 7,2V\% (CLARKE et al., 1996). O controle da concentração anestésica foi obtido por meio de analisador de gases ${ }^{\mathrm{e}}$, cujo sensor foi adaptado à extremidade da sonda orotraqueal, conectado ao circuito anestésico.

Após 30 minutos do início da anestesia inalatória, foi administrada aos animais do GI, buprenorfina $^{\mathrm{f}}$ na dose de $0,02 \mathrm{mg} \mathrm{kg}^{-1}$, pela via intravenosa (IV) enquanto nos animais do grupo GII aplicou-se o opióide na mesma dose utilizada em GI porém pela via intramuscular (IM). Durante toda a fase 
experimental a temperatura dos animais foi mantida estável pelo uso de colchão térmicog.

Foram avaliados a frequiência cardíaca

(FC), obtida em monitor de eletrocardiografia computadorizado $^{\text {h }}$, pelo cálculo do intervalo R-R; pressões arteriais sistólica, média e diastólica (PAS, PAM e PAD, respectivamente), obtidas pela cateterização da artéria femoral esquerda ou direita, cuja leitura foi feita em monitor digital computadorizadoi; débito cardíaco (DC), obtido pela técnica de termodiluição após introdução do cateter de Swan-Ganz pela veia femoral (esquerda ou direita) até o correto posicionamento na artéria pulmonar, cuja leitura foi obtida através do mesmo monitor utilizado para pressão arterial; pressão venosa central (PVC) obtida utilizando-se o conduto do cateter de SwanGanz utilizado para administrar fluido a baixas temperaturas o qual foi previamente posicionado na veia cava ou átrio, segundo técnica descrita por NUNES (2002) e pressão da artéria pulmonar (PAP), obtida pelo mesmo cateter utilizado para o DC. O registro da PVC e da PAP foi feito através do monitor digital utilizado para mensurar a pressão arterial.

As mensurações foram feitas nos seguintes momentos: M1 - 30 minutos após o início da anestesia inalatória, antes da aplicação da buprenorfina; M2 15 minutos após a administração do opióide; M3, M4 e M5 - de 15 em 15 minutos após M2. A análise estatística das variáveis foi efetuada por meio de Análise de Perfil (MORRISON, 1967; CURI, 1980), considerando um nível de significância de 5\% $(\mathrm{p}<0,05)$.

\section{RESULTADOS}

A administração da buprenorfina (M2) determinou, em ambos os grupos, reduções nas variáveis cardiovasculares com maior ênfase no GI. Durante todo o período experimental estes valores permaneceram, no GI, abaixo dos registrados inicialmente, porém dentro dos limites considerados fisiológicos para a espécie. Dentre os parâmetros estudados apenas a PAS e a PAP tiveram diferenças significativas entre os grupos, em M1.

Em relação à FC (Tabela 1), pôde-se observar em GI um acentuado decréscimo de seus valores após M2 $(92,6 \pm 9,57)$, quando comparado a seus números iniciais $(\mathrm{M} 1=118,2 \pm 12,12)$. Houve uma estabilidade dos dados após a queda registrada inicialmente (M2 à M5). No GII, não foram observadas diferenças estatísticas entre os momentos.

O mesmo comportamento de redução pôde ser observado no GI quando se analisaram os dados referentes às pressões arteriais (Tabela 1), ou seja M1 $(\mathrm{PAS}=125,38 \pm 12,13 ; \mathrm{PAD}=71,13 \pm 14,53 ; \mathrm{PAM}=$ $92,00 \pm 14,64$; e PAP $=16,25 \pm 1,49)$ para aqueles a partir de M2 $(\mathrm{PAS}=110,63 \pm 11,75 ; \mathrm{PAD}=57,63 \pm$ $8,33 ; \mathrm{PAM}=77,00 \pm 10,11 ; \mathrm{e} \mathrm{PAP}=14,63 \pm 1,41)$. A PAD continuou a manifestar tendências a queda, após M2, a qual foi estatisticamente significativa em M3 $(56,8 \pm 8,28)$ e M5 $(55,0 \pm 6,11)$.

O DC e a PVC seguiram a mesma tendência de redução registrada em GI para as variáveis citadas anteriormente. Houve, de modo semelhante ao ocorrido com os parâmetros anteriores, estabilização dos valores, não apresentando alteração estatisticamente significativa. Não foi possível identificar quaisquer variações significativas para estas variáveis no GII.

\section{DISCUSSÃO}

A seleção da via de administração de qualquer agente farmacológico deve levar em consideração, além dos aspectos físico-químicos do agente, a facilidade de administração, bem como o tempo necessário para a manifestação de seus efeitos (FLORIO, 1996). Entretanto, outros fatores devem ser avaliados quando da escolha da via de administração, ao se tratar do uso de agentes anestésicos e/ou analgésicos. A necessidade de prever a manifestação de alterações cardiovasculares durante procedimentos anestésicos é fator preponderante para o bom êxito da técnica anestésica.

A buprenorfina, fármaco analgésico opióide com potência superior à da morfina, ainda é pouco utilizada em protocolos anestésicos de pequenos animais. Porém, a manifestação de poucas alterações cardiovasculares associadas à sua longa duração de ação, entre 4 a 8 horas (GADES et al., 2001; PADDLEFORD, 2001) tende a tornar promissora a inclusão deste opióide em tais protocolos. Em contrapartida, a via pela qual este fármaco poderá ser empregado (IV ou IM) implica conhecimentos a respeito da intensidade dos efeitos cardiovasculares decorrentes de tal uso.

$\mathrm{Na}$ avaliação dos parâmetros cardiovasculares propostos, pôde-se observar que estes estavam dentro de níveis considerados fisiológicos para a espécie estudada, antes da aplicação do agente opióide. Tanto a FC como as pressões sistólica, diastólica, média, e da artéria pulmonar, não manifestaram intensamente as ações simpatomiméticas típicas do desfluorano (CLARKE et al., 1996). Tal estabilidade cardiovascular resultou em manutenção do débito cardíaco e da pressão venosa central, não 
Tabela 1 - Valores médios e desvios padrão (entre parênteses) das variáveis cardiovasculares de cães medicados com buprenorfina pelas vias intravenosa (GI) ou intramuscular (GII) durante anestesia com desfluorano.

\begin{tabular}{|c|c|c|c|c|c|c|}
\hline Variável & Grupo & M1 & M2 & M3 & M4 & M5 \\
\hline \multirow{2}{*}{$\mathrm{FC}$ (bat./min.) } & GI & $\begin{array}{c}118,2 \mathrm{a} \\
(12,12)\end{array}$ & $\begin{array}{l}92,6 \mathrm{~b} \\
(9,57)\end{array}$ & $\begin{array}{c}94,2 \mathrm{ab} \\
(9,58)\end{array}$ & $\begin{array}{c}96,6 \mathrm{ab} \\
(9,73)\end{array}$ & $\begin{array}{l}94,7 \mathrm{abc} \\
(11,49)\end{array}$ \\
\hline & GII & $\begin{array}{c}128,3 \\
(16,18)\end{array}$ & $\begin{array}{l}111,2 \\
(23,0)\end{array}$ & $\begin{array}{c}107 \\
(24,31)\end{array}$ & $\begin{array}{c}107,3 \\
(16,93)\end{array}$ & $\begin{array}{c}107,7 \\
(16,35)\end{array}$ \\
\hline \multirow{2}{*}{ PAS (mmHg) } & GI & $\begin{array}{l}125,3 * \\
(14,87)\end{array}$ & $\begin{array}{c}110,6 \\
(11,74)\end{array}$ & $\begin{array}{c}108,7 \\
(11,90)\end{array}$ & $\begin{array}{c}110,7 \\
(13,34)\end{array}$ & $\begin{array}{l}107,0 \\
(9,78)\end{array}$ \\
\hline & GII & $\begin{array}{c}102,2 \\
(23,23)\end{array}$ & $\begin{array}{c}98,3 \\
(16,96)\end{array}$ & $\begin{array}{c}104,1 \\
(16,76)\end{array}$ & $\begin{array}{c}101,2 \\
(15,55)\end{array}$ & $\begin{array}{c}101,8 \\
(14,80)\end{array}$ \\
\hline \multirow{2}{*}{ PAD (mmHg) } & GI & $\begin{array}{c}71,1 \mathrm{a} \\
(14,52)\end{array}$ & $\begin{array}{c}57,6 \\
(8,33)\end{array}$ & $\begin{array}{l}56,8 \mathrm{~b} \\
(8,28)\end{array}$ & $\begin{array}{c}56,8 \\
(8,85)\end{array}$ & $\begin{array}{c}55,0 \mathrm{bc} \\
(6,11)\end{array}$ \\
\hline & GII & $\begin{array}{c}63,2 \\
(8,36)\end{array}$ & $\begin{array}{c}58,8 \\
(12,67)\end{array}$ & $\begin{array}{c}56,0 \\
(9,10)\end{array}$ & $\begin{array}{c}54,5 \\
(7,28)\end{array}$ & $\begin{array}{c}54,3 \\
(7,70)\end{array}$ \\
\hline \multirow{2}{*}{ PAM (mmHg) } & GI & $\begin{array}{c}92 \\
(14,63)\end{array}$ & $\begin{array}{c}77 \\
(10,11)\end{array}$ & $\begin{array}{c}76,6 \\
(9,98)\end{array}$ & $\begin{array}{c}77,6 \\
(10,34)\end{array}$ & $\begin{array}{c}74,7 \\
(8,17)\end{array}$ \\
\hline & GII & $\begin{array}{c}76,3 \\
(18,37)\end{array}$ & $\begin{array}{c}70,6 \\
(15,35)\end{array}$ & $\begin{array}{c}73,3 \\
(13,40)\end{array}$ & $\begin{array}{c}72,1 \\
(11,81)\end{array}$ & $\begin{array}{c}71,7 \\
(11,42)\end{array}$ \\
\hline \multirow{2}{*}{ DC (L/min.) } & GI & $\begin{array}{c}2,05 \\
(0,38)\end{array}$ & $\begin{array}{c}1,84 \\
(0,34)\end{array}$ & $\begin{array}{c}1,96 \\
(0,46)\end{array}$ & $\begin{array}{c}1,92 \\
(0,35)\end{array}$ & $\begin{array}{c}1,85 \\
(0,23)\end{array}$ \\
\hline & GII & $\begin{array}{c}2,32 \\
(0,74)\end{array}$ & $\begin{array}{c}2,21 \\
(0,75)\end{array}$ & $\begin{array}{c}2,18 \\
(0,64)\end{array}$ & $\begin{array}{c}2,32 \\
(0,79)\end{array}$ & $\begin{array}{c}2,26 \\
(0,72)\end{array}$ \\
\hline \multirow{2}{*}{ PVC (mmHg) } & GI & $4,25(1,28)$ & $\begin{array}{c}4,00 \\
(1,51)\end{array}$ & $\begin{array}{c}4,12 \\
(1,45)\end{array}$ & $\begin{array}{c}3,5 \\
(1,19)\end{array}$ & $\begin{array}{c}3,75 \\
(1,38)\end{array}$ \\
\hline & GII & $\begin{array}{c}3,12 \\
(2,23)\end{array}$ & $\begin{array}{c}3,5 \\
(2,97)\end{array}$ & $\begin{array}{c}3,75 \\
(2,81)\end{array}$ & $\begin{array}{c}3,87 \\
(2,35)\end{array}$ & $\begin{array}{c}3,75 \\
(2,76)\end{array}$ \\
\hline \multirow[b]{2}{*}{ PAP (mmHg) } & GI & $\begin{array}{l}16,2^{*} \\
(1,48)\end{array}$ & $\begin{array}{c}14,6 \\
(1,40)\end{array}$ & $\begin{array}{c}14,1 \\
(1,80)\end{array}$ & $\begin{array}{c}15 \\
(1,77)\end{array}$ & $\begin{array}{c}14,6 \\
(1,59)\end{array}$ \\
\hline & GII & $\begin{array}{c}13,7 \\
(1,66)\end{array}$ & $\begin{array}{c}13,6 \\
(3,02)\end{array}$ & $\begin{array}{c}13,8 \\
(2,69)\end{array}$ & $\begin{array}{c}13,6 \\
(2,66)\end{array}$ & $\begin{array}{c}13,6 \\
(2,26)\end{array}$ \\
\hline
\end{tabular}

Para cada variável, médias seguidas pela mesma letra (na linha) ou símbolo (na coluna), não diferem significativamente pela Análise de Perfil ( $\mathrm{p}<0,05$ ). Os símbolos representam a igualdade entre os grupos e as letras a igualdade entre os momentos dentro de cada grupo.

FC - freqüência cardíaca; PAS, PAM e PAD - pressão arterial sistólica, média e diastólica, respectivamente; DC - débito cardíaco; PVC pressão venosa central; PAP - pressão da artéria pulmonar.

sendo registradas reduções dignas de nota, conforme o descrito na literatura (PAGEL et al., 1991).

A queda da FC observada em ambos os grupos após a administração da buprenorfina (M2), demonstra sua ação cronotrópica negativa, podendo ser proveniente de efeito vagotônico central (STEPIEN et al., 1995; MARTINEZ et al., 1997). Uma avaliação mais detalhada destes dados (Tabela 1) possibilitou verificar que a magnitude deste efeito cronotrópico foi mais intenso no GI, não sendo significativo no GII. Tal achado sugere que a administração por via IV possibilita ao fármaco se encontrar sob a forma livre no sangue numa velocidade superior à da via IM, fazendo com que este rapidamente alcance os órgãos mais perfundidos (FLORIO, 1996).
No tocante às pressões arteriais sistólica e média (PAS e PAM), pôde-se notar que não ocorreram alterações clínicas significativas ao longo do período anestésico, apesar de a partir de M2 as variações terem sido mais intensas no GI, porém não significativas pelo teste estatístico empregado. É interessante ressaltar que a PAD foi a variável que teve declínio mais acentuado, em ambos os grupos, a qual poderia estar diretamente relacionada à diminuição da resistência vascular periférica (GANONG, 1993). Este efeito foi considerado significativo estatisticamente no GI, com queda mais acentuada 30 minutos após a administração do opióide (M3). Esta redução pode estar relacionada à maior biodisponibilidade do fármaco (TAYLOR et al., 2001) o que tornaria mais intensos os seus efeitos. 
Com relação à PAP se evidenciou apenas um discreto declínio do seu valor inicial, após a administração do opióide com maior ênfase no GI, sem significado clínico ou estatístico. Tal redução é compatível com aquela observada na PAM (Tabela 1), pois a PAP tem estreita relação com a pressão arterial sistêmica, sendo 6 a 8 vezes mais baixa que esta (FRANCHINI, 1999). A permanência deste parâmetro em patamares fisiológicos permitiu a manutenção do trabalho cardíaco direito e a conseqüente troca gasosa pulmonar (WEST, 1990).

Quanto ao DC, o uso da buprenorfina determinou uma redução dos valores, novamente com maior intensidade em GI, apesar de não ter sido estatisticamente significativo. Como o DC é produto da freqüência cardíaca pelo volume sistólico (DOWNEY, 2000), pode-se afirmar que tal achado foi obtido em função da redução da FC provocada pelo opióide (STEPIEN et al., 1995), bem como por uma provável redução da força contrátil do miocárdio. Outrossim, é válido relacionar uma possível redução do retorno venoso ao coração, determinado pelo opióide, o qual, apesar de ter sido discreto, poderia, junto com a redução da FC e da contratilidade do miocárdio determinar queda do DC, pois segundo DOWNEY (2000) o retorno venoso e o DC têm que ser iguais. Este autor ainda cita, corroborando tais afirmativas, o fato de que quanto mais o coração é distendido na diástole, maior é a força de sua contração, relação conhecida como lei de Frank-Starling.

Pôde-se constatar que a PVC seguiu a tendência manifestada nas demais variáveis cardiovasculares, ou seja, queda após a administração da buprenorfina, a qual foi mais intensa aos 45 minutos após a sua aplicação. Este efeito pode estar relacionado à diminuição do DC, promovida pelo desfluorano (PAGEL et al., 1991), associado à diminuição da FC e da pressão arterial sistêmica, promovidas pela buprenorfina (STEPIEN et al., 1995), com eventual redução na pré-carga. Interessante ressaltar que esta tendência de queda não se manifestou no GII, exatamente pela menor intensidade de alterações nas variáveis citadas anteriormente.

\section{CONCLUSÃo}

Com base nos resultados obtidos, pode-se assumir como hipótese conclusiva que os efeitos decorrentes do uso da buprenorfina pelas vias IV ou IM são similares não apresentando diferenças marcantes nos parâmetros cardiovasculares estudados. Entretanto, estudos complementares deverão ser realizados em indivíduos portadores de distúrbios cardiovasculares para melhor determinar a participação destas alterações nesta classe de pacientes.

\section{AGRADECIMENTO}

Os autores agradecem à Fundação de Amparo à Pesquisa no Estado de São Paulo (FAPESP) pelo apoio financeiro concedido.

\section{FONTE DE AQUISIÇÃO}

a - PROVIVE - Zeus Lifesciences Ltda., São Paulo - SP. b - SUPRANE - ZENECA Farmacêutica do Brasil Ltda. c - OHMEDA - mod. Excel 210SE - Datex Ohmeda, Miami EUA

d - OHMEDA - mod. TEC 6 - Datex Ohmeda, Miami - EUA e - OHMEDA - mod. 5220 - Datex Ohmeda, Miami - EUA f - TEMGESIC - Schering-Plough S.A., Rio de Janeiro - RJ. g - GAYMAR - Model TP500 - Gaymar Inc., Londres - ING. h - TEB - Model ECGPC 1.10 - Tecnologia Eletrônica Brasileira, São Paulo - SP

i - DIXTAL DX 2010 - DIXTAL Biomédica Ind. Com. Ltda., São Paulo - SP

\section{REFERÊNCIAS BIBLIOGRÁFICAS}

CLARKE, K.W. et al. Cardiopulmonary effects of desflurane in the dog during spontaneous and artificial ventilation. Res Vet Sc, v.61, p.82-86, 1996.

CURI, P.R. Análise de medidas repetidas em experimentos biológicos. Rev Bras Estat, v.41, p.137-150, 1980.

DOWNEY, J.M. Regulação do retorno venoso e do débito cardíaco. In: JOHNSON, L.R. Fundamentos de fisiologia médica. 2.ed. Rio de Janeiro : Guanabara Koogan, 2000. p.169-175.

EGER, E.I II. Desflurane animal and human pharmacology: aspects of kinetics, safety, and MAC. Anesth Analg, v.75, p.3-9, 1992.

FLORIO, J.C. Absorção, distribuição, biotransformação e eliminação. In: SPINOSA, H. S.; BERNARDI, M.M.; GÓRNIAK, S.L. (Eds.). Farmacologia aplicada à medicina veterinária. Rio de Janeiro : Guanabara Koogan, 1996. p.23-37.

FRANCHINI, K.G. Circulações regionais. In: AIRES, M.M Fisiologia. 2.ed. Rio de Janeiro : Guanabara Koogan, 1999. Cap.40. p.448-472.

GADES, N.M. et al. The magnitude and duration of the analgesic effect of morphine, butorphanol, and buprenorphine in rats and mice. Contemp Top Lab Anim Sci, v.40, n.2, p.8-13, 2001

GANONG, W.F. Dinâmica do sangue e do fluxo linfático. In: - Fisiologia médica. Rio de Janeiro : Prentice-Hall do Brasil, 1993. p.408-420.

GÓRNIAK, S.L. Hipnoanalgésicos e neuroleptoanalgesia. In: SPINOSA, H.S.; BERNARDI, M.M.; GÓRNIAK, S.L. (Eds.). Farmacologia aplicada à medicina veterinária. Rio de Janeiro: Guanabara Koogan, 1996. Cap.15, p.140-146.

MARTINEZ, E.A. et al. Cardiovascular effects of buprenorphine in anesthetized dogs. Am J Vet Res, v.58, n.11, p.1280-1283, 1997. 
MORRISON, D.F. Multivariate statistical methods. New York: McGrows Hill, 1967. 388p.

NOVALIJA, E. et al. Effects of desflurane, sevoflurane and halothane on postinfarction spontaneous dysrhythmias in dogs. Acta Anaesthesiol Scand, v.42, n.3, p.353-357, 1998.

NUNES, N. Monitoração da anestesia. In: FANTONI, D.T.; CORTOPASSI, S.R.G. Anestesia em cães e gatos. São Paulo: Rocca, 2002. Cap.6, p.64-81.

PACENTINE, G.G; MUZI, M.; EBERT, T.J. Effects of fentanyl on sympathetic activation associated with the administration of desflurane. Anest, v.82, p.823-831, 1995.

PADDLEFORD, R.R. Analgesia e controle da dor. In:

Manual de anestesia em pequenos animais. 2.ed. São Paulo: Roca, 2001. p.263-285.

PAGEL, P.S. et al. Influence of volatile anesthetics on myocardial contractily in vivo: desflurane versus isoflurane. Anest, v.74, p.900-907, 1991.

PRECKEL, B. et al. Effects of enflurane, isoflurane, sevoflurane and desflurane on reperfusion injury after regional myocardial ischaemia in the rabbit heart in vivo. Br J Anaesth, v.81, n.6, p.905-912, 1998.

SANTOS, P.S.P. et al. Eletrocardiografia de cães submetidos a diferentes concentrações de desfluorano, pré-tratados ou não com a associação de fentanil-droperidol. Ciênc Rural, v.31, n.5, p.805811, 2001.

SMILEY, R.M. An overview of induction and emergence characterictics of desflurane in pediatric, adult, and geriatric patients. Anesth Analg, v.75, p.38-46, 1992.

STEPIEN, R.L. et al. Cardiorespiratory effects of acepromazine maleate and buprenorphine hydrochloride in clinically normal dogs. Am J Vet Res, v.56, n.1, p.78-84, 1995.

TAYLOR, P.M. et al. Morphine, pethidine and buprenorphine disposition in the cat. J Vet Pharm Therap, v.24, n.6, p.391400, 2001.

THURMON, J.C.; TRANQUILLI, W.J.; BENSON, G.J. Injectable anesthetics. In:___. Lumb \& Jones' veterinary anesthesia. 3.ed. Philadelphia: Lea \& Feabiger, 1996. Chapt.9, p.210-240.

WARLTIER, D.C., PAGEL, P.S. Cardiovascular and respiratory actions of desflurane: is desflurane different from isoflurane? Anesth Analg, v.75, p.17-31, 1992.

WEISKOPF, R.B. et al. Desflurane does not produce hepatic or renal injury in human volunteers. Anesth Analg, v.74, p.570574, 1992.

WEST, J.B. Pulmonary blood flow and metabolism. In: Best and Taylor'S physiological basis of medical practice. 12.ed. Baltimore : Williams \& Wilkins, 1990. Chapt.36, p.529-537. 